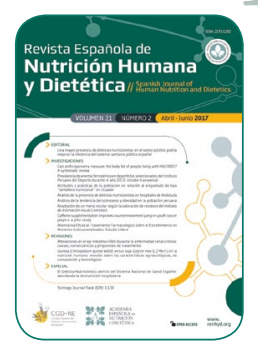

\title{
REVISIÓN
}

\section{Alteraciones en el eje intestino-riñón durante la enfermedad renal crónica: causas, consecuencias y propuestas de tratamiento}

\author{
Iván Armando Osuna-Padilla ${ }^{a, *}$, Gabriela Leal-Escobar ${ }^{b}$ \\ ${ }^{a}$ Centro de Investigación en Enfermedades Infecciosas, Instituto Nacional de Enfermedades Respiratorias, México. \\ bClínica de Diálisis Peritoneal, Instituto Nacional de Cardiología "Ignacio Chávez", México. \\ *ivan.osuna@cieni.org.mx
}

Recibido el 18 de mayo de 2016; aceptado el 8 de febrero de 2017; publicado el 9 de mayo de 2017.

\author{
PALABRAS CLAVE \\ Insuficiencia Renal \\ Crónica; \\ Microbioma \\ Gastrointestinal; \\ Uremia; \\ Probióticos; \\ Prebióticos; \\ Terapia Dietética.
}

Alteraciones en el eje intestino-riñón durante la enfermedad renal crónica: causas, consecuencias y propuestas de tratamiento

\section{RESUMEN}

La enfermedad renal crónica y el estado urémico se asocian con alteraciones en la permeabilidad intestinal y cambios en la microbiota intestinal, provocando una mayor producción y translocación de toxinas urémicas como sulfato de indoxilo (IS) y sulfato de p-cresilo (pCS), detonando una respuesta inflamatoria. El estado inflamatorio y el incremento en concentraciones séricas de IS y pCS se han asociado con una mayor mortalidad, mayor número de eventos cardiovasculares y mayores alteraciones en el metabolismo mineral y óseo. Se han estudiado diversas estrategias nutricionales y farmacológicas para modular la microbiota intestinal y mejorar las alteraciones en la permeabilidad intestinal, entre ellas la suplementación con probióticos, prebióticos y simbióticos, modificaciones en la composición de la dieta y uso de agentes adsorbentes. El objetivo del presente trabajo es realizar una revisión de las causas de las alteraciones intestinales y de la microbiota intestinal en el paciente con enfermedad renal crónica, analizando las consecuencias de dichos cambios y las intervenciones estudiadas hasta la actualidad. 
Kidney-gut axis disruption in chronic kidney disease: causes, consequences and treatment strategies

\section{KEYWORDS}

Renal Insufficiency, Chronic;

Gastrointestinal

Microbiome;

Uremia;

Probiotics;

Prebiotics;

Diet Therapy.

\section{ABSTRACT}

Chronic kidney disease and uremic state are associated with intestinal barrier permeability alterations and changes in gut microbiome composition that increased the production and translocation of uremic toxins such indoxyl sulfate (IS) and p-cresyl sulfate (pCS) and contribute to the immunological response. Uremic toxins and inflammation are associated with and increase on mortality, cardiovascular events and mineral bone disorders. Several nutritional and pharmacological strategies have been studied to modulate the gut microbiota composition and intestinal permeability alterations, including probiotic, prebiotic and symbiotic supplementation, changes in diet composition and use of adsorbent agents. The aim of this study is to review the etiology of intestinal and microbiota alterations analyzing the consequences and interventions studied to date.

\section{CITA}

Osuna-Padilla IA, Leal-Escobar G. Alteraciones en el eje intestino-riñón durante la enfermedad renal crónica: causas, consecuencias y propuestas de tratamiento. Rev Esp Nutr Hum Diet. 2017; 21(2): 174-83. doi: 10.14306/ renhyd.21.2.244

\section{INTRODUCCIÓN}

Diversas condiciones presentes en el paciente con enfermedad renal crónica (ERC) se asocian al desarrollo de alteraciones en la permeabilidad de la barrera intestinal y en la microbiota intestinal, entre las que destacan la pérdida de la función renal, la toxicidad urémica, el uso frecuente de antibióticos, el consumo disminuido de fibra en la alimentación y la baja ingestión de líquidos ${ }^{1}$.

La permeabilidad intestinal se ve comprometida debido a una disminución en la expresión de las proteínas que conforman las uniones estrechas intestinales, provocando con ello translocación de microorganismos y de antígenos alimentarios ${ }^{1}$.

La microbiota intestinal sufre cambios en su composición, incrementando las familias con capacidad para sintetizar urea, ácido úrico y otros compuestos como los indoles y fenoles, los cuales traspasan la barrera intestinal debido a la disfuncionalidad de la misma ${ }^{1}$.
Ambas condiciones desencadenan una respuesta inmunológica que se ha asociado con una mayor mortalidad y mayor incidencia de eventos cardiovasculares ${ }^{1}$.

El objetivo del presente trabajo es realizar una revisión de las causas de las alteraciones intestinales y de la microbiota intestinal en el paciente con ERC, analizando las consecuencias de dichos cambios y las intervenciones no farmacológicas estudiadas hasta la actualidad.

Para cumplir dicho objetivo, se realizó una búsqueda bibliográfica del 1 de abril al 1 de mayo de 2016, identificando las publicaciones posteriores al 2010 de las bases de datos on-line PubMed/Medline, Scielo y LILACS. Como criterios de inclusión se consideraron a los estudios de revisión, estudios descriptivos y estudios de intervención. No fueron incluidos estudios publicados en forma de resumen o actas de congresos, así como aquellos publicados en un idioma distinto al inglés y al español. Como palabras clave se utilizaron: enfermedad renal crónica, microbiota intestinal, uniones estrechas e inflamación. 


\section{$\longrightarrow$ PERMEABILIDAD INTESTINAL}

El mantenimiento de la barrera epitelial intestinal es fundamental para evitar la translocación de antígenos y bacterias presentes en el lumen intestinal. Su integridad depende del mantenimiento del balance de enterocitos, así como de las uniones presentes entre dichas células, denominadas uniones estrechas (del inglés, tight junction), cuyas estructuras proteicas forman fibrillas que atraviesan la membrana plasmática e interactúan con las proteínas de las células aledañas. De forma intracelular, las proteínas interactúan con el anillo de actomiosina que rodea el enterocito. Dichas proteínas consisten en dos tipos de trepaspaninas, denominadas ocludina y claudina, las cuáles interactúan en el citoplasma con la familia de proteínas denominadas zonula occludens, la cual está compuesta por tres variantes (ZO-1, ZO-2 y ZO-3). La interacción entre dichas proteínas forma una barrera entre una célula y otra, protegiendo al organismo del paso de toxinas y microorganismos, regulando también el transporte paracelular de iones, agua y otros solutos².

La funcionalidad de estas uniones se ve alterada por diversos factores, entre ellos, los procesos inflamatorios, la calidad de la dieta, la motilidad intestinal y la presencia de ERC1, según se ha observado en modelos animales urémicos. Éstos cursan con una disminución marcada en las proteínas que conforman las uniones estrechas en colon (70-90\% en claudina-1, 50-70\% en ocludina y 80-90\% en Z0-1), teniendo por consecuencia la translocación bacteriana ${ }^{3}$. La disminución en dichas proteínas se ha observado también en distintos sitios del tracto gastrointestinal en humanos (estómago, yeyuno e íleon) ${ }^{4}$. Se han identificado distintos mecanismos causantes de la disminución de dichas proteínas, destacando el incremento en las concentraciones de urea en el intestino, hidrolizándose a hidróxido de amonio, el cual ocasiona erosión de la barrera intestinal y disminución en la resistencia eléctrica transepitelial. La erosión de la barrera intestinal ocasiona la estimulación de leucocitos, produciendo citoquinas inflamatorias de manera local, provocando retracción y endocitosis de las proteínas mencionadas ${ }^{5,6}$.

\section{ENDOTOXEMIA Y ACTIVACIÓN INMUNE}

La forma más común de evaluar la translocación bacteriana es analizando las concentraciones séricas de endotoxina. La endotoxina, también denominada lipopolisacárido (LPS), es un componente de la membrana de microorganismos gramnegativos, los cuales representan el $70 \%$ de los microorganismos residentes del lumen intestinal, correspondientes a aproximadamente 500-1000 especies bacterianas distintas. Las concentraciones séricas de LPS se incrementan en presencia de infección, y en ausencia de ésta, las concentraciones elevadas (endotoxemia) se asocian a una translocación bacteriana incrementada, a consecuencia de disfunción intestinal. La endotoxemia ha sido ampliamente descrita en pacientes urémicos con ERC, asociándose a la presencia de infecciones recurrentes en los catéteres en pacientes bajo tratamiento renal sustitutivo, a periodontitis, a la contaminación de las soluciones dializantes, al estado de sobrehidratación, y recientemente a las alteraciones en las uniones estrechas intestinales ${ }^{7-10}$.

EI LPS, al ser un patrón molecular asociado a microorganismos patógenos, ocasiona la activación del sistema inmunológico innato, al entrar en contacto con diversas proteínas, entre las que destacan la proteína de unión a LPS (LBP, por sus siglas en inglés), receptores CD14 y Toll 4 (TLR4). La proteína LBP capta el LPS circulante, formando un complejo LPS-LBP, el cual permite la asociación de LPS con el receptor CD14, cuya función es activar al TLR4; el complejo encargado de su reconocimiento. La activación del TLR4 conlleva la activación de dos factores de transcripción; proteína activadora (AP-1) y factor nuclear kB (NF-kB) ${ }^{11}$. Al activarse dichas vías se estimula la producción de citoquinas proinflamatorias, entre ellas prostaglandina 2 (PGE2), factor de necrosis tumoral (TNF- $\alpha$ ), interleucina 1 (IL-1), interleucina 6 (IL-6), interferón (IFN) y proteína $C$ reactiva. Ésta inflamación de etiología intestinal se suma a la inflamación crónica ocasionada por la pérdida de la función renal y las complicaciones de la misma, entre ellas la acidosis metabólica y el estrés oxidativo ${ }^{12}$.

La activación inmunológica ha sido estudiada por Raj y Cols., al observar en una cohorte de 310 pacientes con ERC en hemodiálisis, una asociación positiva entre las concentraciones de la proteína CD14 soluble (sCD14) con las citoquinas proinflamatorias TNF- $\alpha(r=0,24 ; p<0,001)$ e IL-6 $(r=0,18$; $p=0,002)$, observando una mayor mortalidad en los pacientes con concentraciones más altas de sCD14 (RR 1,94; $\mathrm{p}=0,04)^{13}$.

La inflamación crónica en la ERC ha sido relacionada con un mayor riesgo para las siguientes condiciones: eventos cardiovasculares, aterosclerosis temprana, desgaste proteicoenergético, alteraciones en el metabolismo mineral óseo y anemia resistente a eritropoyetina ${ }^{14}$. 


\section{$\longrightarrow$ DISBIOSIS INTESTINAL Y PRODUCCIÓN DE TOXINAS URÉMICAS}

En los últimos años la microbiota intestinal ha sido objeto de estudio por su papel en los procesos de salud y enfermedad, describiéndose diversas funciones, entre ellas su interacción con el sistema inmunológico y sistema nervioso central, además de distintas implicaciones en procesos metabólicos ${ }^{15}$.

Denominada el "órgano olvidado"16, la microbiota intestinal está conformada por más de 1012 UFC/mL, compuesta por cerca de 1000 especies de bacterias distintas, dominadas por cuatro filos: Actinobacteria, Proteobacteria, Bacteroidetes y Firmicutes, estos dos últimos conforman más del $90 \%$ del total de las bacterias intestinales ${ }^{17}$. La disbiosis intestinal, definida como la alteración en la composición de la microbiota intestinal, está presente en diversas enfermedades crónicas no transmisibles, entre ellas la diabetes mellitus tipo 2, la obesidad y la $\mathrm{ERC}^{17,18}$.

Vaziri y Cols. han descrito la presencia de cambios en la composición de la microbiota intestinal en individuos con ERC, al comparar a 24 adultos con ERC y 12 adultos sanos, observaron diferencias significativas en más de 190 unidades taxonómicas operacionales de microorganismos (UTOs), principalmente de los géneros y familias Brachybacterium, Catenibacterium, Enterobacteriaceae, Halomonadaceae, Moraxellaceae, Nesterenkonia, Polyangiaceae, Pseudomonadaceae y Thiotrix. Estos hallazgos fueron corroborados en ratones, en quienes indujeron ERC al someterlas a nefrectomía, describiendo diferencias en 175 UTOs bacterianos entre los ratones urémicos y controles ${ }^{19}$.

Dichos cambios obedecen a una expansión de las familias bacterianas que poseen enzimas ureasa (Alteromonadaceae, Cellulomonadaceae, Clostridiaceae, Dermabacteraceae, Enterobacteriaceae, Halomonadaceae, Methylococcaceae, Micrococcaceae, Moraxellaceae, Polyangiaceae, Pseudomonadaceae y Xanthomonadaceae), uricasa (Cellulomonadaceae, Dermabacteraceaea, Micrococcaceae, Polyangiaceae y Xanthomonadacea) y de algunas familias de microorganismos que tienen la capacidad para formar indol y $p$-cresol (Clostridiaceae, Enterobacteriaceae y Verrucomicrobiaceae). Dicha expansión se acompaña de una disminución en el número de microorganismos con capacidad para utilizar la fibra y formar ácidos grasos de cadena corta (Lactobacillaceae y Prevotellaceae), según lo reportado por Wong y Cols ${ }^{20}$.
No existe hasta el momento una causalidad única que explique los cambios reportados. Se han propuesto diversas hipótesis etiológicas, entre ellas que el cambio es producto del incremento en el flujo de urea y ácido úrico al intestino ${ }^{21}$. Otra de las hipótesis es el posible impacto de la restricción de líquidos y de alimentos ricos en potasio, disminuyendo con ello el consumo de frutas y verduras principalmente, las cuales son fuentes importantes de fibra. Kalantar-Zadeh y Cols. reportaron en el 2001 un consumo promedio de fibra de $17 \mathrm{~g} /$ día en pacientes con ERC sometidos a hemodiáli$\operatorname{sis}^{22,23}$. La disminución en el consumo de ésta puede contribuir a la disminución de las familias de microorganismos con capacidad para su utilización, las cuales son productoras de ácidos grasos de cadena corta ${ }^{24}$.

Aronov y Cols. han demostrado que la expansión de dichas familias en el paciente con ERC tiene por consecuencia la producción de toxinas urémicas, al comparar las muestras de plasma de 10 individuos sanos, 9 individuos con ERC sometidos a hemodiálisis cuyo colon estaba intacto y 6 individuos con ERC sometidos a hemodiálisis con colectomía total $^{25}$. En sus resultados, demuestran que los individuos con ERC y colon intacto tienen una mayor cantidad de toxinas urémicas comparado con el grupo sometido a colectomía, describiendo la formación colónica de p-cresol e sulfato de indoxilo (IS, por sus siglas en inglés). Así mismo, evaluaron los cambios de dichas toxinas antes y después de la sesión de hemodiálisis, encontrando que éstas no eran removidas efectivamente por dicha terapia, elucidando así el papel de la microbiota intestinal en la formación de solutos urémicos y su contribución en el síndrome urémico ${ }^{25}$. La Figura 1 describe las alteraciones en el eje intestino-riñón presentes en el paciente con ERC.

Las toxinas urémicas son generadas por la microbiota intestinal al metabolizar algunos precursores presentes en la dieta. Las familias más estudiadas en la actualidad son los fenoles, indoles, hipuratos y el $\mathrm{N}$-óxido de trimetilamina $(\mathrm{TMAO})^{26}$, reportándose una relación inversa entre la tasa de filtrado glomerular y las concentraciones séricas de IS $(r=-0,72 ; p<0,001)$ y de $p$-cresil sulfato $(r=-0,64 ; p<0,001)$, asociación que describe el papel del riñón en la eliminación fisiológica de las mismas ${ }^{27}$.

EI TMAO es un metabolito tóxico producido por el intestino, cuyo precursor es la L-carnitina y la fosfatidilcolina dietética, la cual es convertida en trimetilamina por la microbiota intestinal, para posteriormente absorberse y convertirse en TMAO por la monooxigenasa en el hígado ${ }^{28}$. Sus concentraciones se relacionan de forma directa con el consumo de alimentos de origen animal, específicamente de carne roja y huevo ${ }^{29}$. 
La desaminación de aminoácidos aromáticos ocasiona la producción de compuestos fenólicos; la tirosina produce fenol y p-cresol, la fenilalanina produce fenilacetato, y el triptófano produce indolacetato e indol, el cual es metabolizado en el hígado produciendo IS. El p-cresol es convertido a p-cresil sulfato (pCS) por la enzima sulfotransferasa citoplasmática y liberado a la circulación ${ }^{30}$ (Figura 2).

Figura 1. Causas y consecuencias de las alteraciones en el eje intestino-riñón durante la ERC.

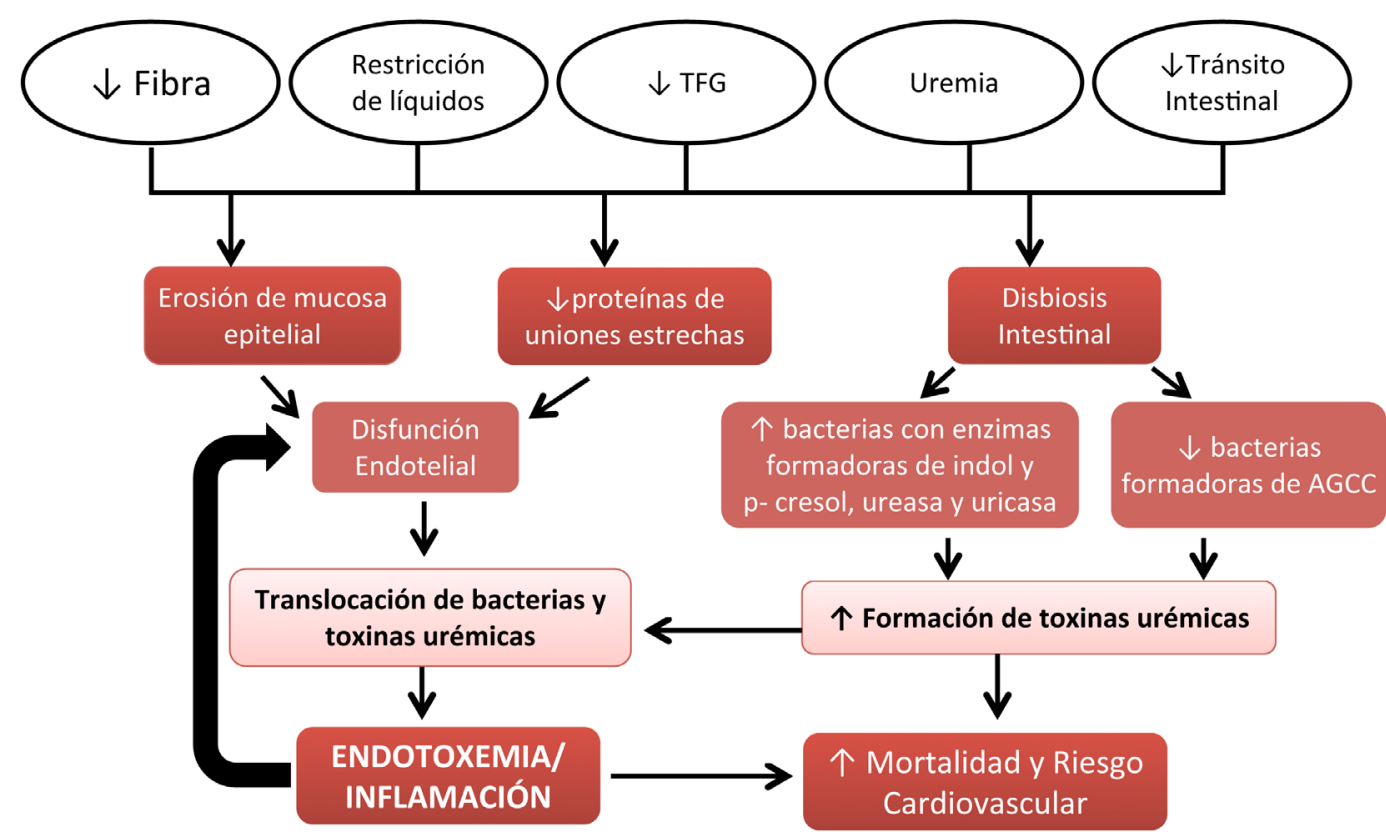

TFG: Tasa de Filtrado Glomerular; AGCC: Ácidos Grasos de Cadena Corta.

Figura 2. Producción de Toxinas Urémicas y Consecuencias en el Organismo.

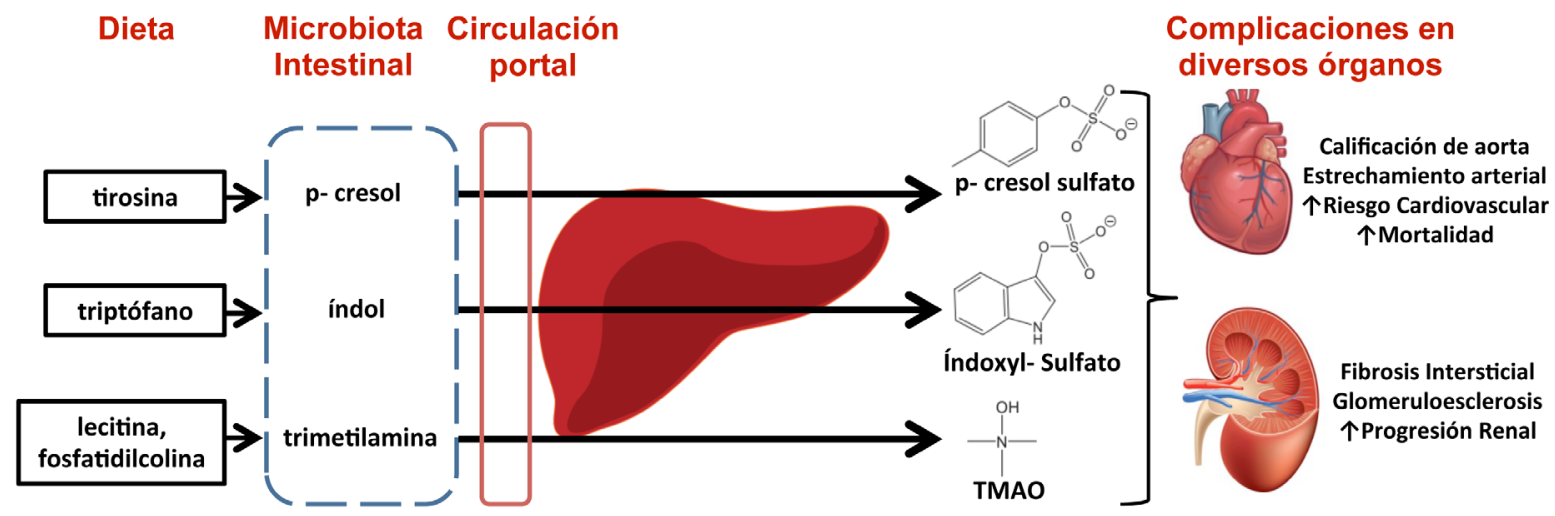

TMAO: N-óxido de trimetilamina. 


\section{TOXINAS URÉMICAS Y RIESGO CARDIOVASCULAR}

La pérdida de la función renal y el proceso inflamatorio que acompaña la enfermedad condicionan un estado patológico con implicaciones graves en el sistema cardiovascular, siendo la primera causa de muerte en los pacientes con ERC en terapia renal sustitutiva ${ }^{27}$.

La disminución en la función renal resulta en la retención de diferentes compuestos que normalmente deberían ser excretados en orina. Mientras algunos compuestos son removidos por la terapia dialítica, un gran número de sustancias no lo son, tal es el caso del IS y pCS, que al ser toxinas urémicas unidas a proteínas (albúmina), no son eliminadas, incrementado sus concentraciones en plasma ${ }^{27}$. Se ha observado un incremento en los valores séricos hasta 54 veces mayor para pCS y 17 veces mayor para IS en comparación con la población sana ${ }^{31}$.

Las concentraciones elevadas de pCS e IS se asocian con una mayor mortalidad, según los resultados de un metaanálisis de 11 estudios prospectivos publicado por Lin y Cols. ${ }^{31}$, quienes reportan una mayor mortalidad en pacientes con concentraciones séricas elevadas de pCS (OR=1,16; IC95\%:1,03-1,30; $\mathrm{p}=0,013)$ e IS (OR=1,10; IC95\%:1,03-1,17; $p=0,003)$ y mayor riesgo de eventos cardiovasculares en pacientes con $\mathrm{pCS}$ elevado (OR=1,28; IC95\%:1,10-1,50; $p=0,002)$. Las concentraciones elevadas se han asociado también con la progresión de la ERC, según lo reportado por Wu y Cols., quienes observaron en su estudio que valores elevados de IS y $p$ cresol se asocian con una progresión acelerada de la enfermedad, independientemente el grado de $\mathrm{ERC}^{32}$.

El mecanismo exacto de cómo los valores séricos de pCS e IS contribuyen a la presencia de eventos cardiovasculares e incrementan la mortalidad no ha sido del todo elucidado. Se documenta que ambas toxinas tienen un efecto proinflamatorio, el cuál altera la función endotelial mediante diferentes mecanismos, provocando estrés oxidativo en las células endoteliales, alterando los mecanismos de reparación de las células endoteliales, además de incrementar la proliferación de células del músculo liso vascular. En lo que respecta a la progresión de la enfermedad, en modelos animales ambas toxinas urémicas se han asociado con daño en los túbulos renales, induciendo fibrosis intersticial y glomeruloesclero$\mathrm{sis}^{33}$.

Se ha documentado también que las concentraciones elevadas de IS y pCS tienen un impacto deletéreo en la salud ósea, al disminuir la respuesta de CAMP (adenosín monofosfato cíclico) a la hormona paratiroidea, inhibir la diferenciación de osteoclastos y producir daño a células osteoblásticas, al incrementar la producción y activación de anticuerpos específicos para su fosforilación, incrementando con ello la resorción ósea y exacerbando las alteraciones en el metabolismo mineral y óseo característico de la ERC ${ }^{34}$.

De igual manera, las concentraciones séricas de TMAO se han asociado con riesgo cardiovascular, aunque a diferencia de $\mathrm{pCS}$ e IS, éste sí es removido de forma eficiente durante la terapia dialítica ${ }^{35}$. Wilson-Tang y Cols. describieron en una cohorte de 521 pacientes con ERC sin tratamiento renal sustitutivo, una asociación entre las concentraciones elevadas de TMAO y el riesgo de infarto de miocardio, accidente cerebrovascular y muerte en los próximos 5 años ( $R R=2,76$; $p<0,001)^{36}$. En modelos animales, se reporta una asociación entre las concentraciones elevadas de TMAO con una mayor fibrosis tubulointersticial y deposición de colágeno, como resultado de la fosforilación de Smad3, el cual es un regulador de la vía de señalización TFG-B/Smad3, la cual es profibróti$\mathrm{ca}$, contribuyendo así a la disfunción renal progresiva ${ }^{36}$.

\section{Estrategias terapéuticas}

Conociendo las alteraciones intestinales presentes en la ERC y sus consecuencias, se han estudiado diversas estrategias terapéuticas con capacidad para modular la composición de la microbiota intestinal y el sobrecrecimiento de microorganismos (probióticos, prebióticos, simbióticos y modificaciones dietéticas), y disminuir la absorción de toxinas urémicas en el intestino (agentes adsorbentes) ${ }^{27}$.

Los probióticos son definidos como microorganismos vivos que cuando se administran en cantidades adecuadas confieren un beneficio a la salud del hospedador ${ }^{37}$. Entre los beneficios reportados tras su utilización destacan: 1) el mantenimiento de la integridad intestinal, al funcionar como una barrera física para los microorganismos patógenos, confiriendo una resistencia a la colonización de los mismos; 2 ) la prevención de infecciones al producir algunos péptidos antimicrobianos o bacteriocinas, eliminando con ello algunos patógenos y estimulando al sistema inmunológico innato; 3) la potenciación del sistema inmunológico innato y adaptativo, al tener la habilidad para promover la diferenciación de células B e incrementar la producción de lgA; y 4) la modulación de la respuesta inflamatoria, al promover un mejor control en la secreción de citoquinas proinflamatorias ${ }^{33,38}$.

Estudios realizados en pacientes con ERC han tenido por objetivo evaluar el efecto de la suplementación de probióticos en las concentraciones de toxinas urémicas. Entre los resultados destacan la disminución de IS y pCS en plasma 
y de otras toxinas urémicas (dimetilamina y nitrosodimetilamina $)^{38}$. Otro de los objetivos ha sido evaluar el impacto en la respuesta inflamatoria, observando disminución en TNF- $\alpha$, IL- 6 y LPS en 46 pacientes en diálisis peritoneal tras 6 meses de suplementación con Bifidobacterium bifidum, B. catenulatum, $B$. longum y Lactobacillus plantarum ${ }^{39}$. Se ha descrito también una disminución en proteína $C$ reactiva e indoxil glucorónido en 22 pacientes en hemodiálisis tras 6 meses de suplementación con Streptococcus thermophilus, L. acidophilus y B. longum, sin alcanzar significancia estadística ${ }^{40}$. Miranda-Alatriste y Cols. reportaron una disminución significativa en las concentraciones de urea sanguínea tras la suplementación de la cepa L. casei Shirota en dosis de $16 \times 10^{9}$ UFC en 30 individuos con ERC grado 3 y 4 sin tratamiento renal sustitutivo ${ }^{41}$.

Los prebióticos son definidos como ingredientes no digeribles de los alimentos que afectan beneficiosamente al huésped estimulando selectivamente el crecimiento y/o la actividad de una de las especies de bacterias que ya están establecidas en el colon, o de un número limitado de ellas y, por consiguiente, mejoran la salud del huésped ${ }^{37}$.

La suplementación con prebióticos se ha utilizado como estrategia para aminorar el síndrome urémico, al contribuir a una mayor excreción de nitrógeno en las heces fecales, además de disminuir la producción de toxinas urémicas en el intestino. Entre los prebióticos más estudiados en el paciente con ERC se encuentra la inulina de agave, los fructooligosacáridos y los galactooligosacáridos ${ }^{42}$.

Se ha documentado la disminución de IS tras suplementar fibra ( $15 \mathrm{~g}$ de almidón resistente) durante 6 semanas en 56 individuos en hemodiálisis, sin cambios en el pCS ${ }^{43}$, resultados que coinciden con un metaanálisis de Rossi y Cols., quienes no observaron disminución en pCS al suplementar fibra ${ }^{44}$. Recientemente, se ha descrito en otro metaanálisis de 14 ensayos clínicos la disminución significativa en las concentraciones de urea y creatinina en pacientes con ERC con y sin terapia sustitutiva tras la suplementación de fibra (media de duración 4,5 semanas, $86 \%$ de los estudios utilizaban fibra soluble, dosis promedio $26,9 \mathrm{~g} /$ día ${ }^{45}$.

Algunos autores han utilizado mezclas de prebióticos y probióticos, los cuales se denominan simbióticos. Guida y Cols. evaluaron el efecto de un simbiótico ( 9 especies de probióticos, $2,2 \mathrm{~g}$ de inulina y $1,3 \mathrm{~g}$ de almidón resistente) por 4 semanas en las concentraciones de pCS y en la sintomatología gastrointestinal de 30 pacientes con ERC estadio 3-4, observando una disminución significativa en esta toxina urémica en el grupo que recibió el tratamiento comparado con el grupo control, no observándose cambios en sintomatología gastrointestinal ${ }^{46}$. Viramontes-Hörner y Cols. sí observaron mejorías en la sintomatología gastrointestinal en 21 pacientes en hemodiálisis, sin observar cambios significativos en marcadores inflamatorios, tras 2 meses de suplementación con un producto que contenía $L$. acidophilus y $B$. animalis subesp. lactis, $2,3 \mathrm{~g}$ de inulina y $1,5 \mathrm{~g}$ de omega-347. Este mismo grupo de trabajo reportó cambios en la composición de la microbiota intestinal, con incrementos en la cuenta de bifidobacterias tras 2 meses de suplementación del mismo producto en 18 pacientes en terapia hemodiálisis ${ }^{48}$. Estos hallazgos han sido replicados por Rossi y Cols., quienes reportan una disminución en pCS, con incrementos en bifidobacterias tras suplementar por 6 semanas $15 \mathrm{~g}$ de fibra en polvo (inulina, fructooligosacáridos y galactooligosacáridos) y una cápsula con 9 diferentes cepas de lactobacilos, bifidobacterias y estreptococos en 31 individuos con ERC grado 4-549.

La dieta del individuo con ERC es un factor que repercute en la producción de toxinas urémicas dependiendo el tipo de aminoácidos consumidos. Se ha estudiado el impacto de una alimentación baja en proteínas y del uso de alfacetoanálogos de aminoácidos en la producción de toxinas urémicas $^{29}$. Marzocco y Cols. reportaron que la instauración de una dieta muy baja en proteínas $(0,3 \mathrm{~g} / \mathrm{kg} /$ día) suplementada con alfacetoanálogos de aminoácidos por una semana, disminuye en un $37 \%$ las concentraciones de IS en individuos sin terapia sustitutiva ${ }^{50}$. Estos resultados han sido debatidos, ya que Rossi y Cols. no observaron una correlación entre el consumo de proteínas y las concentraciones séricas de pCS e IS. Este grupo de autores propone la utilización de un indicador denominado índice de proteína/fibra, el cual se correlaciona con las concentraciones séricas de IS $(r=0,40$; $p=0,012)$ y $p C S(r=0,43 ; p=0,005)^{51}$.

El enfoque dietético centrado en proveer la cantidad óptima de macronutrientes y micronutrientes, y no centrado únicamente en la restricción de proteínas, puede proveer de algunos otros beneficios metabólicos, como mejorar el control glucémico y las cifras de tensión arterial. Debido a ello, se ha propuesto la utilización del patrón dietético mediterráneo (DMED) en el paciente con ERC, confiriendo los beneficios al tipo de hidratos de carbono y las bajas cantidades de proteína de origen animal que conlleva dicho patrón alimentario. Se han propuesto algunos beneficios de la DMED en la composición de la microbiota intestinal, al favorecer el tránsito intestinal por las fuentes de fibra que incluye y disminuir con ello la producción de toxinas urémicas ${ }^{52}$, sin embargo su efectividad es controversial. En el estudio multicéntrico PREDIMED, Díaz-López y Cols. no observaron un beneficio en la función renal de la dieta mediterránea frente a una dieta control, tras 1 año de seguimiento en pacientes con alto riesgo de enfermedad cardiovascular sin diagnóstico de $E R C^{53}$. Por su parte, Huang y Cols. reportaron una menor incidencia de ERC en pacientes con una mayor adherencia a 
la dieta mediterránea (OR=0,59; IC95\%:0,38-0,87; $p=0,04)$ medida con la herramienta Mediterranean Diet Score, en un estudio transversal en 1.110 adultos mayores de 70 años en Suiza ${ }^{54}$. A la fecha, no se han localizado ensayos clínicos que evalúen el efecto de la misma en población con diagnóstico de ERC establecido. La dieta mediterránea es alta en potasio y fósforo, por lo que su indicación en pacientes con una tasa de filtrado $<60 \mathrm{~mL} / \mathrm{min} / 1,73^{2}$ puede tener efectos deletéreos, al no excretarse de forma adecuada estos nutrimentos. Se necesitan estudios que evalúen la seguridad en dichos grados de la ERC, así como el impacto en la producción de toxinas urémicas.

Tomando en cuenta el posible impacto de los aminoácidos aromáticos en la producción de toxinas urémicas, se ha propuesto la utilización de la dieta vegetariana como estrategia para reducir la producción de toxinas urémicas. Patel y Cols. reportaron una menor excreción de pCS (-62\%) e IS (-58\%) en población sin ERC que llevaban una alimentación vegetariana, lo cual podría explicarse posiblemente por tener un mayor consumo de fibra y un menor consumo de proteínas respecto a la población que sigue una dieta con fuentes de origen animal $\left.\right|^{55}$. Estos hallazgos fueron replicados recientemente en pacientes con ERC en hemodiálisis por Kandouz y Cols., quienes observaron menores concentraciones séricas de pCS e IS en pacientes vegetarianos estrictos comparados con pacientes omnívoros ${ }^{56}$.

En lo que respecta a estrategias no dietéticas, se ha propuesto la utilización de agentes con capacidad de adsorber las toxinas urémicas en el lumen intestinal, e impedir con ello su absorción. Schulman ha estudiado ampliamente la eficacia de un adsorbente oral (AST-120) en la ERC, el cual consiste en microesferas de un material de carbón poroso, encontrando que su suplementación restaura las proteínas de las uniones estrechas, disminuye la endotoxina plasmática y los marcadores de estrés oxidativo e inflamación en modelos animales con ERC, mostrando una disminución dosis-dependiente en las concentraciones de IS tras suplementar por 12 semanas diferentes dosis $\left(0,9 ; 2,1\right.$ y 3,0g) tres veces al día en pacientes con $E R C^{57}$. No se ha logrado demostrar que la inclusión de este agente a la terapia conservadora retrase la progresión de la ERC ${ }^{58}$.

\section{CONCLUSIONES}

La uremia ocasiona alteración en las uniones estrechas intestinales, ocasionando disfunción de la barrera epitelial, al disminuir la expresión de las proteínas que conforman dichos sitios. Asimismo, ocasiona disbiosis intestinal, incrementándose las familias de microorganismos con capacidad para formar toxinas urémicas. La disfunción intestinal ocasiona translocación de microorganismos patógenos al torrente circulatorio. La translocación de microorganismos y de toxinas urémicas producidas por la microbiota intestinal se asocia con una respuesta inflamatoria crónica, asociándose con mayor mortalidad, eventos cardiovasculares y alteraciones en el metabolismo mineral y óseo. Se ha estudiado el uso de probióticos, no existiendo consenso sobre las cepas a utilizar en pacientes con ERC. La utilización de prebióticos ha mostrado efectividad en la reducción de IS, pero no existe consenso respecto a qué tipo y cantidad de fibra habría que suplementar al paciente con ERC. El uso de simbióticos para estos pacientes, no queda claro, ya que los efectos observados no pueden ser adjudicados únicamente al simbiótico, además de que los estudios analizados para la presente revisión presentan tamaños muestrales pequeños. La utilización de una dieta baja en proteínas suplementada con alfacetoanálogos de aminoácidos ha mostrado tener un impacto positivo en la producción de IS, pero no todos los pacientes son candidatos a recibir este tipo de suplementación, además de los costos económicos que implica su prescripción; sin embargo, no se ha observado una asociación positiva entre el consumo de proteínas en la dieta y las concentraciones de pCS e IS. No se han localizado estudios que hayan evaluado la utilización de la dieta mediterránea en pacientes con ERC, el impacto en la producción de toxinas urémicas y el efecto en las concentraciones de fósforo y potasio. La dieta vegetariana ha mostrado disminuir la producción de pCS e IS en individuos con y sin ERC, sin embargo, hacen falta estudios que corroboren dichos beneficios, considerando el tamaño muestral de las publicaciones halladas. El uso de agentes adsorbentes puede ser efectivo para disminuir las concentraciones de IS plasmático.

\section{CONFLICTO DE INTERESES}

Los autores expresan que no hay conflictos de interés al redactar el manuscrito.

\section{REFERENCIAS}

(1) Kotanko P, Carter M, Levin NW. Intestinal bacterial microflora-a potential source of chronic inflammation in patients with chronic kidney disease. Nephrol Dial Transplant. 2006; 21(8): 2057-60. 
(2) Günzel D, Yu ASL. Claudins and the modulation of tight junction permeability. Physiol Rev. 2013; 93(2): 525-69.

(3) Vaziri ND, Yuan J, Rahimi A, Ni Z, Said H, Subramanian VS. Disintegration of colonic epithelial tight junction in uremia: a likely cause of CKD-associated inflammation. Nephrol Dial Transplant. 2012; 27(7): 2686-93.

(4) Vaziri ND, Yuan J, Nazertehrani S, Ni Z, Liu S. Chronic kidney disease causes disruption of gastric and small intestinal epithelial tight junction. Am J Nephrol. 2013; 38(2): 99-103.

(5) Vaziri ND, Yuan J, Norris K. Role of urea in intestinal barrier dysfunction and disruption of epithelial tight junction in chronic kidney disease. Am J Nephrol. 2013; 37(1): 1-6.

(6) Vaziri ND, Goshtasbi N, Yuan J, Jellbauer S, Moradi H, Raffatellu $M$, et al. Uremic plasma impairs barrier function and depletes the tight junction protein constituents of intestinal epithelium. Am J Nephrol. 2012; 36(5): 438-43.

(7) Gonçalves S, Pecoits-Filho R, Perreto S, Barberato SH, Stinghen AEM, Lima EGA, et al. Associations between renal function, volume status and endotoxaemia in chronic kidney disease patients. Nephrol Dial Transplant. 2006; 21(10): 2788-94.

(8) McIntyre CW, Harrison LEA, Eldehni MT, Jefferies HJ, Szeto C-C, John SG, et al. Circulating endotoxemia: a novel factor in systemic inflammation and cardiovascular disease in chronic kidney disease. Clin J Am Soc Nephrol. 2011; 6(1): 133-41.

(9) Hauser AB, Stinghen AEM, Gonçalves SM, Bucharles S, Pecoits-Filho R. A gut feeling on endotoxemia: causes and consequences in chronic kidney disease. Nephron Clin Pract. 2011; 118(2): c165-172; discussion c172.

(10) Machowska A, Carrero J], Lindholm B, Stenvinkel P. Therapeutics targeting persistent inflammation in chronic kidney disease. Transl Res. 2016; 167(1): 204-13.

(11) de Punder K, Pruimboom L. Stress induces endotoxemia and low-grade inflammation by increasing barrier permeability. Front Immunol. 2015; 6: 223.

(12) Mafra D, Fouque D. Gut microbiota and inflammation in chronic kidney disease patients. Clin Kidney J. 2015; 8(3): 332-4.

(13) Raj DSC, Carrero JJ, Shah VO, Qureshi AR, Bárány P, Heimbürger 0 , et al. Soluble CD14 levels, interleukin 6, and mortality among prevalent hemodialysis patients. Am J Kidney Dis. 2009; 54(6): 1072-80.

(14) Akchurin OM, Kaskel F. Update on inflammation in chronic kidney disease. Blood Purif. 2015; 39(1-3): 84-92.

(15) Gerritsen J, Smidt H, Rijkers GT, de Vos WM. Intestinal microbiota in human health and disease: the impact of probiotics. Genes Nutr. 2011; 6(3): 209-40.

(16) Clemente JC, Ursell LK, Parfrey LW, Knight R. The impact of the gut microbiota on human health: an integrative view. Cell. 2012; 148(6): 1258-70.

(17) Villanueva-Millán MJ, Pérez-Matute P, Oteo JA. Gut microbiota: a key player in health and disease. A review focused on obesity. ] Physiol Biochem. 2015; 71(3): 509-25.

(18) Guinane CM, Cotter PD. Role of the gut microbiota in health and chronic gastrointestinal disease: understanding a hidden metabolic organ. Therap Adv Gastroenterol. 2013; 6(4): 295-308.

(19) Vaziri ND, Wong J, Pahl M, Piceno YM, Yuan J, DeSantis TZ, et al. Chronic kidney disease alters intestinal microbial flora. Kidney Int. 2013; 83(2): 308-15.

(20) Wong J, Piceno YM, Desantis TZ, Pahl M, Andersen GL, Vaziri ND. Expansion of urease- and uricase-containing, indole- and p-cresol-forming and contraction of short-chain fatty acidproducing intestinal microbiota in ESRD. Am J Nephrol. 2014; 39(3): 230-7.

(21) Vaziri ND. CKD impairs barrier function and alters microbial flora of the intestine: a major link to inflammation and uremic toxicity. Curr Opin Nephrol Hypertens. 2012; 21(6): 587-92.

(22) Kalantar-Zadeh K, Kopple JD, Deepak S, Block D, Block G. Food intake characteristics of hemodialysis patients as obtained by food frequency questionnaire. J Ren Nutr. 2002; 12(1): 17-31.

(23) Aron-Wisnewsky J, Clément K. The gut microbiome, diet, and links to cardiometabolic and chronic disorders. Nat Rev Nephrol. 2016; 12(3): 169-81.

(24) Evenepoel P, Meijers BK. Dietary fiber and protein: nutritional therapy in chronic kidney disease and beyond. Kidney Int. 2012; 81(3): 227-9.

(25) Aronov PA, Luo FJ-G, Plummer NS, Quan Z, Holmes S, Hostetter $\mathrm{TH}$, et al. Colonic contribution to uremic solutes. ] Am Soc Nephrol. 2011; 22(9): 1769-76.

(26) Vanholder R, Glorieux G. The intestine and the kidneys: a bad marriage can be hazardous. Clin Kidney J. 2015; 8(2): 168-79.

(27) Meijers BKI, Evenepoel P. The gut-kidney axis: indoxyl sulfate, p-cresyl sulfate and CKD progression. Nephrol Dial Transplant. 2011; 26(3): 759-61.

(28) Vaziri ND, Zhao Y-Y, Pahl MV. Altered intestinal microbial flora and impaired epithelial barrier structure and function in CKD: the nature, mechanisms, consequences and potential treatment. Nephrol Dial Transplant. 2016; 31(5): 737-46.

(29) Moraes C, Fouque D, Amaral ACF, Mafra D. Trimethylamine N-Oxide From Gut Microbiota in Chronic Kidney Disease Patients: Focus on Diet. J Ren Nutr. 2015; 25(6): 459-65.

(30) Mafra D, Lobo JC, Barros AF, Koppe L, Vaziri ND, Fouque D. Role of altered intestinal microbiota in systemic inflammation and cardiovascular disease in chronic kidney disease. Future Microbiol. 2014; 9(3): 399-410.

(31) Lin C-J, Wu V, Wu P-C, Wu C-J. Meta-Analysis of the Associations of p-Cresyl Sulfate (PCS) and Indoxyl Sulfate (IS) with Cardiovascular Events and All-Cause Mortality in Patients with Chronic Renal Failure. PLoS ONE. 2015; 10(7): e0132589.

(32) Wu I-W, Hsu K-H, Lee C-C, Sun C-Y, Hsu H-J, Tsai C-J, et al. $\mathrm{p}$-Cresyl sulphate and indoxyl sulphate predict progression of chronic kidney disease. Nephrol Dial Transplant. 2011; 26(3): 938-47.

(33) Ramezani A, Raj DS. The gut microbiome, kidney disease, and targeted interventions. J Am Soc Nephrol. 2014; 25(4): 657-70.

(34) Black AP, Cardozo LFMF, Mafra D. Effects of Uremic Toxins from the Gut Microbiota on Bone: A Brief Look at Chronic Kidney Disease. Ther Apher Dial. 2015; 19(5): 436-40.

(35) Tang WHW, Wang Z, Levison BS, Koeth RA, Britt EB, Fu X, et al. Intestinal microbial metabolism of phosphatidylcholine and cardiovascular risk. N Engl J Med. 2013; 368(17): 1575-84.

(36) Tang WHW, Wang Z, Kennedy DJ, Wu Y, Buffa JA, Agatisa-Boyle $B$, et al. Gut microbiota-dependent trimethylamine $\mathrm{N}$-oxide (TMAO) pathway contributes to both development of renal insufficiency and mortality risk in chronic kidney disease. Circ Res. 2015; 116(3): 448-55.

(37) World Health Organization, Food and Agriculture Organization of the United Nations. Probiotics in food: Health and nutritional properties and guidelines for evaluation. Roma, Italia: FAO/WHO; 2006. 
(38) Koppe L, Mafra D, Fouque D. Probiotics and chronic kidney disease. Kidney Int. 2015; 88(5): 958-66.

(39) Wang I-K, Wu Y-Y, Yang Y-F, Ting I-W, Lin C-C, Yen T-H, et al. The effect of probiotics on serum levels of cytokine and endotoxin in peritoneal dialysis patients: a randomised, double-blind, placebo-controlled trial. Benef Microbes. 2015; 6(4): 423-30.

(40) Natarajan R, Pechenyak B, Vyas U, Ranganathan P, Weinberg $A$, Liang $P$, et al. Randomized controlled trial of strain-specific probiotic formulation (Renadyl) in dialysis patients. Biomed Res Int. 2014; 2014: 568571.

(41) Miranda Alatriste PV, Urbina Arronte R, Gómez Espinosa CO, Espinosa Cuevas $M$ de los Á. Effect of probiotics on human blood urea levels in patients with chronic renal failure. Nutr Hosp. 2014; 29(3): 582-90.

(42) Rossi M, Johnson DW, Campbell KL. The Kidney-Gut Axis: Implications for Nutrition Care. J Ren Nutr. 2015; 25(5): 399-403.

(43) Sirich TL, Plummer NS, Gardner CD, Hostetter TH, Meyer TW. Effect of increasing dietary fiber on plasma levels of colon-derived solutes in hemodialysis patients. Clin J Am Soc Nephrol. 2014; 9(9): 1603-10.

(44) Rossi M, Klein K, Johnson DW, Campbell KL. Pre-, pro-, and synbiotics: do they have a role in reducing uremic toxins? A systematic review and meta-analysis. Int J Nephrol. 2012; 2012: 673631.

(45) Chiavaroli L, Mirrahimi A, Sievenpiper JL, Jenkins DJA, Darling PB. Dietary fiber effects in chronic kidney disease: a systematic review and meta-analysis of controlled feeding trials. Eur ] Clin Nutr. 2015; 69(7): 761-8.

(46) Guida B, Germanò R, Trio R, Russo D, Memoli B, Grumetto L, et al. Effect of short-term synbiotic treatment on plasma p-cresol levels in patients with chronic renal failure: a randomized clinical trial. Nutr Metab Cardiovasc Dis. 2014; 24(9): 1043-9.

(47) Viramontes-Hörner D, Márquez-Sandoval F, Martín-del-Campo F, Vizmanos-Lamotte B, Sandoval-Rodríguez A, ArmendárizBorunda J, et al. Effect of a symbiotic gel (Lactobacillus acidophilus + Bifidobacterium lactis + inulin) on presence and severity of gastrointestinal symptoms in hemodialysis patients. J Ren Nutr. 2015; 25(3): 284-91.

(48) Cruz-Mora J, Martínez-Hernández NE, Martín del Campo-López F, Viramontes-Hörner D, Vizmanos-Lamotte B, Muñoz-Valle
JF, et al. Effects of a symbiotic on gut microbiota in Mexican patients with end-stage renal disease. J Ren Nutr. 2014; 24(5): 330-5.

(49) Rossi M, Johnson DW, Morrison M, Pascoe EM, Coombes JS, Forbes JM, et al. Synbiotics Easing Renal Failure by Improving Gut Microbiology (SYNERGY): A Randomized Trial. Clin ] Am Soc Nephrol. 2016; 11(2): 223-31.

(50) Marzocco S, Dal Piaz F, Di Micco L, Torraca S, Sirico ML, Tartaglia $D$, et al. Very low protein diet reduces indoxyl sulfate levels in chronic kidney disease. Blood Purif. 2013; 35(1-3): 196-201.

(51) Rossi M, Johnson DW, Xu H, Carrero JJ, Pascoe E, French C, et al. Dietary protein-fiber ratio associates with circulating levels of indoxyl sulfate and p-cresyl sulfate in chronic kidney disease patients. Nutr Metab Cardiovasc Dis. 2015; 25(9): 860-5.

(52) Montemurno E, Cosola C, Dalfino G, Daidone G, De Angelis $M$, Gobbetti $M$, et al. What would you like to eat, Mr CKD Microbiota? A Mediterranean Diet, please! Kidney Blood Press Res. 2014; 39(2-3): 114-23.

(53) Díaz-López A, Bulló M, Martínez-González MÁ, Guasch-Ferré M, Ros E, Basora J, et al. Effects of Mediterranean diets on kidney function: a report from the PREDIMED trial. Am J Kidney Dis. 2012; 60(3): 380-9.

(54) Huang X, Jiménez-Moleón J], Lindholm B, Cederholm T, Arnlöv J, Risérus $U$, et al. Mediterranean diet, kidney function, and mortality in men with CKD. Clin J Am Soc Nephrol. 2013; 8(9): 1548-55.

(55) Patel KP, Luo FJ-G, Plummer NS, Hostetter TH, Meyer TW. The production of $p$-cresol sulfate and indoxyl sulfate in vegetarians versus omnivores. Clin J Am Soc Nephrol. 2012; 7(6): 982-8.

(56) Kandouz S, Mohamed AS, Zheng Y, Sandeman S, Davenport A. Reduced protein bound uraemic toxins in vegetarian kidney failure patients treated by haemodiafiltration. Hemodial Int. 2016; 20(4): 610-7.

(57) Schulman G, Vanholder R, Niwa T. AST-120 for the management of progression of chronic kidney disease. Int ] Nephrol Renovasc Dis. 2014; 7: 49-56.

(58) Schulman G, Berl T, Beck GJ, Remuzzi G, Ritz E, Arita K, et al. Randomized Placebo-Controlled EPPIC Trials of AST-120 in CKD. J Am Soc Nephrol. 2015; 26(7): 1732-46. 